\title{
CONTROL GERENCIAL: EL ANÁLISIS DEL COSTEO VARIABLE Y ABSORBENTE COMO HERRAMIENTA DE GESTIÓN
}

LIZARDO AGÜERO DEL CARPIO*

E-mail: lizaguero@yahoo.com

RESUMEN

Las Organizaciones al encontrarse en un mundo altamente competitivo y de turbulencia, están optando en mirarse hacia adentro, en una actitud introspectiva, obviamente, sin descuidar su atención al cliente, o lo que se conoce como la Función Relacional con el Cliente o el Customer Relationshing Managemente (CRM).

Por eso es de vital importancia, conocer las Estructura Interna de los Costos de las organizaciones, y el manejo de las mismas, los cuales evidentemente repercutirán en el precio y en la rentabilidad de las organizaciones. Es así que las Empresas están optando por herramientas de planificación en el manejo de los costos, como es el tratamiento de los costos directos o variables, herramienta valiosa que incluye en la estructura de los costos, solo aquellos costos o erogaciones variables en la fabricación del producto o prestación de servicios.

Los costos por absorción, que incluyen en la estructura de los costos tanto los costos variables como los fijos, en la determinación de los costos unitarios de producción.

Palabras clave: Sistema de costos: por absorción, directo, variable y fijo.

\section{ABSTRACT}

The Organizations, being in a competitive and turbulent world, are opting for looking toward inside, in an introspective attitude, obviously, without neglecting their attention to the client, or what is known as the Relational Function with the Client of the Customer Relationship Management (CMR).

For that reason it is of vital importance, to know the Internal Structure of the Costs of the organizations, and the handling of the same ones, those which evidently will rebound in the price and in the profitability of the organizations. It is so the treatment of the direct or variable costs, valuable tool that includes in the structure of the costs only those costs or variable expenditures in the production of the product or benefit of services.

The costs for absorption that include the structure of the costs are both the variable costs and the fixed ones, in determining the unitary costs of production

Keywords: System of costs: for absorption, direct, variable and fix.

* Lic. en Administración de Empresas y Contador Público Colegiado. Magíster en Banca y Finanzas. Docente Universitario de la Facultad de Ciencias Administrativas; UNMSM. 
Hoy en día la gestión de las empresas debe buscar la máxima eficiencia y efectividad en sus operaciones, razón por la cual toda organización, sea esta de prestación de servicios o de producción de bienes, debe poner énfasis en su estructura de costos y evaluar el impacto financiero de sus decisiones.

En el presente artículo se tratará de explicar cómo se valora la rentabilidad, utilizando los métodos de Costeo Absorbente y de Costeo Variable. Luego de comparar estos conceptos, se explicará la forma en que las empresas lo utilizan para controlar:

- Sus costos.

- Determinar el precio de sus productos.

- Planear la producción.

- Analizar segmentos de mercados y

- Estudiar los márgenes de contribución.

\section{EL ESTADO DE RESULTADOS BAJO LA VISIÓN DEL COSTEO VARIABLE O ABSORBENTE Y EL COSTEO ABSORBENTE}

En toda organización ya sea pública, privada y/o mixta, una de las variables que afecta el ingreso neto, es el costo de los bienes vendidos.

El costo de ventas, entendido como el costo de los artículos vendidos (costo de adquisición y/o producción), se puede determinar tomando como base el método del costeo absorbente o el método del costeo variable.

\section{Costeo absorbente}

Se considera como costeo absorbente al costeo por medio del cual todos los productos fabricados se absorben hacia los productos terminados y permanecen allí como un activo, hasta que estos se vendan o liquiden.

El costeo absorbente es necesario para la determinación de los costos históricos y para los informes financieros dirigido a los usuarios externos (inversionistas, estado, etc.).

\section{Costeo variable}

El costeo variable suele ser mas útil para los procesos de toma de decisiones, también conocido como costeo directo.

En el costeo variable, los costos de la producción solo se componen de costos variables de producción, representando a todos aquellos costos que aumentan o disminuyen, conforme aumenta y crece el volumen de la producción.

Entre estos costos podemos citar a la mano de obra directa, materiales directos, y a aquellos gastos indirectos de fabricación que varían en proporción directa con el nivel de producción; el resto de gastos indirectos de fabricación no forman parte del costo de los productos fabricados y por lo tanto constituyen gastos operativos del periodo.

Consideraremos el siguiente caso a fin de comprender el concepto:

La empresa La Fabricante SAC, cuenta con la siguiente información:

\section{Costo de producción}

\begin{tabular}{|lccc|}
\hline & $\begin{array}{c}\text { S/. Costo } \\
\text { Total }\end{array}$ & $\begin{array}{c}\text { Número de } \\
\text { Unidades }\end{array}$ & $\begin{array}{c}\text { Costo } \\
\text { Unitario }\end{array}$ \\
\hline Costos Variables & 550000,00 & 16500 & 33,33 \\
\hline Costos Fijos & 180000,00 & 16500 & 10,91 \\
\hline Total & 730000,00 & & 44,24 \\
\hline
\end{tabular}

Gastos de ventas y administrativos del periodo

\begin{tabular}{|lr|}
\hline Gastos Variables S/. 28,00 por unidad & S/. \\
\hline Gastos Fijos & 300000,00 \\
\hline Total & 492000,00 \\
\hline
\end{tabular}

Ahora, se sabe que se venden 16500 unidades y cada unidad se vende a un precio de S/. 90 .

Elaboraremos el Estado de Ganancias y pérdidas por el método del costeo variable y por el método de costeo absorbente, y evaluaremos su incidencia en la estructura de los resultados.

Tenemos que tener en cuenta que en el Estado de Ganancias y Pérdidas, por costeo variable, solo incluiremos los costos variables de producción en el costo de los productos vendidos.

En la elaboración del Estado de Ganancias y Pérdidas separaremos los costos variables de los costos fijos. El costo variable de los productos vendidos, que incluyen los costos variables de producción, se deducen de las ventas para dar lugar a la contribución marginal de producción.

Los gastos variables de ventas y administración se deducen de la contribución marginal de producción para obtener una contribución marginal. 


\begin{tabular}{|c|c|}
\hline \multicolumn{2}{|c|}{$\begin{array}{l}\text { Estado de Ganancias y Pérdidas por el Método del } \\
\text { Costeo Variable (Expresado en nuevos soles ) }\end{array}$} \\
\hline (16 500 unidades $\times$ S/. 90,00) & 1485000,00 \\
\hline $\begin{array}{l}\text { Costos variables } \\
\text { de los productos (16 } 500 \text { unidades x S/. 33,33) } \\
\text { Vendidos }\end{array}$ & $(550000,00)$ \\
\hline Margen de producción & 935055,00 \\
\hline Gastos variables de venta y administración & $(462000,00)$ \\
\hline Margen de contribución & 473055,00 \\
\hline \multicolumn{2}{|l|}{ Costos fijos } \\
\hline Costos fijos de producción & \\
\hline Gastos fijos de ventas & \\
\hline Ingresos de operaciones & 263000,00 \\
\hline
\end{tabular}

A continuación presentaremos el Estado de Ganancias y Pérdidas bajo el método absorbente, que no hace distinción entre costos variables y fijos. Nótese que todos los costos de producción incluyen el costo de ventas originando una diferencia sustancial, producto de la deducción de ésta con las ventas, en relación con el método de costeo variable.

\begin{tabular}{|lr|}
\hline \multicolumn{2}{|c|}{$\begin{array}{c}\text { Estado de Ganancias y Pérdidas por el Método del } \\
\text { Costeo Absorbente (Expresado en nuevos soles ) }\end{array}$} \\
\hline Ventas $\quad(16500$ unidades x S/. 90,00) & 1485000,00 \\
\hline $\begin{array}{l}\text { Costos de los } \\
\text { productos vendidos (16 } 500 \text { unidades x S/. 44,24) }\end{array}$ & $(730000,00)$ \\
\hline Utilidad bruta & 755000,00 \\
\hline Gastos de ventas y administración & $(492000,00)$ \\
\hline Margen de contribución & 263000,00 \\
\hline
\end{tabular}

Nótese en el método de costeo variable que el resultado de la Utilidad Bruta representa el margen de contribución, luego de deducidos los costos variables $(\mathrm{S} / .473055,00)$ frente a los S/. 755 000,00 del costo absorbente, lo que no hace distingo alguno de la variabilidad de la producción, factor fundamental en todo proceso de decisión en los procesos de planta. Ahora analizaremos qué sucede cuando las unidades producidas, exceden a las unidades vendidas, en el mismo caso:

\begin{tabular}{|lrr|}
\hline \multicolumn{3}{|c|}{$\begin{array}{c}\text { Estado de Ganancias y Perdidas por el Método del } \\
\text { Costeo Variable (Expresado en nuevos soles) }\end{array}$} \\
\hline Ventas & $(15000$ unidades x S/. 90,00) & 1350000,00 \\
\hline $\begin{array}{l}\text { Costos variables } \\
\text { de los productos } \\
\text { vendidos }\end{array}$ & $(16500$ unidades x S/. 33,33) & $(549$ 945,00) \\
\hline Menos inventario final & $(1500 \times 33,33)$ & $(49995,00)$ \\
\hline Margen de producción & 850050,00 \\
\hline
\end{tabular}

\begin{tabular}{|lrr|}
\hline $\begin{array}{l}\text { Gastos variables de venta } \\
\text { y administración }\end{array}$ & $(15000,00 \times \mathrm{S} / .28,00)$ & $(420000,00)$ \\
\hline Margen de contribución & 430050,00 \\
\hline Costos fijos & & \\
\hline Costos fijos de producción & 180000,00 & \\
\hline Gastos fijos de ventas & 30000,00 & $(210000,00)$ \\
\hline Ingresos de operaciones & 220050,00 \\
\hline
\end{tabular}

\begin{tabular}{|lr|}
\hline \multicolumn{2}{|c|}{$\begin{array}{c}\text { Estado de Ganancias y Pérdidas por el Método del } \\
\text { Costeo Absorbente (Expresado en nuevos soles) }\end{array}$} \\
\hline Ventas $\quad(15000$ unidades x S/. 90.00) & 1350000.00 \\
\hline $\begin{array}{l}\text { Costo de los } \\
\text { productos vendidos (15000 unidades x S/. 44.24) }\end{array}$ & $(663600.00)$ \\
\hline Utilidad bruta & 686400.00 \\
\hline Gastos de ventas y administración & $(450000.00)$ \\
\hline Ingreso de operaciones & 236400.00 \\
\hline
\end{tabular}

Ahora entendemos que al analizar y evaluar las operaciones, la Gerencia debe ser consciente de los posibles efectos de cambiar los niveles de inventario bajo los dos métodos.

Siguiendo con el mismo caso, supongamos que las ventas estimadas son de 20000 unidades a un precio de venta de $\mathrm{S} / .90$, sin importar en absoluto los niveles de producción.

Además se sabe que la compañía evalúa dos probables niveles de producción, así tenemos:

\section{Propuesta I}

Producir y vender 20000 unidades

\section{Costo de producción}

\begin{tabular}{|lccc|}
\hline & $\begin{array}{c}\text { S/. Costo } \\
\text { Total }\end{array}$ & $\begin{array}{c}\text { Número de } \\
\text { Unidades }\end{array}$ & $\begin{array}{c}\text { Costo } \\
\text { Unitario }\end{array}$ \\
\hline Costos variables & 550000,00 & 20000 & 27,50 \\
\hline Costos fijos & 180000,00 & 20000 & 9,00 \\
\hline Total & 730000,00 & & 36,50 \\
\hline
\end{tabular}

Gastos de ventas y administrativos del periodo

\begin{tabular}{|lr|}
\hline Gastos variables S/. 15.00 por unidad & S/. 300000,00 \\
\hline Gastos fijos & 30000,00 \\
\hline Total & 330000,00 \\
\hline
\end{tabular}




\section{Propuesta II}

Producir 25000 unidades y vender 20000 unidades

\section{Costo de producción}

\begin{tabular}{|lccc|}
\hline & $\begin{array}{c}\text { S/. Costo } \\
\text { Total }\end{array}$ & $\begin{array}{c}\text { Número de } \\
\text { Unidades }\end{array}$ & $\begin{array}{c}\text { Costo } \\
\text { Unitario }\end{array}$ \\
\hline Costos variables & 687500,00 & 25000 & 27,50 \\
\hline Costos fijos & 180000,00 & 25000 & 7,20 \\
\hline Total & 887500,00 & & 34,70 \\
\hline
\end{tabular}

Gastos de ventas y administrativos del periodo

\begin{tabular}{|lr|}
\hline Gastos Variables S/. 15,00 por unidad & S/. 375000,00 \\
\hline Gastos Fijos & 30000,00 \\
\hline Total & 330000,00 \\
\hline
\end{tabular}

Ahora determinaremos los efectos de aplicar cada método en el Estado de Ganancias y Pérdidas. Iniciamos con el método del costeo absorbente:

Con 20000 unidades producidas y vendidas:

\begin{tabular}{|lr|}
\hline \multicolumn{2}{|c|}{$\begin{array}{c}\text { Estado de Ganancias y Pérdidas por el Método del } \\
\text { Costeo Absorbente (Expresado en nuevos soles) }\end{array}$} \\
\hline Ventas $\quad(20000$ unidades x S/. 90,00) & 1800000,00 \\
\hline $\begin{array}{l}\text { Costo de los } \\
\text { productos vendidos }(20000 \text { unidades x S/. 36,50) }\end{array}$ & $(730000,00)$ \\
\hline Utilidad bruta & 1070000,00 \\
\hline Gastos de ventas y administración & $(330000,00)$ \\
\hline Ingreso de operaciones & 740000,00 \\
\hline
\end{tabular}

Con 25000 unidades producidas y 20000 vendidas:

\begin{tabular}{|c|c|}
\hline \multicolumn{2}{|c|}{$\begin{array}{l}\text { Estado de Ganancias y Pérdidas por el Método del } \\
\text { Costeo Absorbente (Expresado en nuevos soles) }\end{array}$} \\
\hline$(20000$ unidades $\times$ S/. 90,00) & 1800000,00 \\
\hline $\begin{array}{l}\text { Costo de los } \\
\text { productos vendidos }\end{array}$ & 867500,00 \\
\hline Menos inventario final 5000 x S/. 34,70 (173 500) & $(694000,00)$ \\
\hline Utilidad bruta & 1110600,00 \\
\hline Gastos de ventas y administración & $(330000,00)$ \\
\hline Ingreso de operaciones & 776000,00 \\
\hline
\end{tabular}

Podemos apreciar que los ingresos de las operaciones se han visto incrementados en S/. 36 000,00 (776 000 - 740000 ), produciendo 25000 unidades e incrementando el inventario de productos terminados en 5000 unidades.
El aumento en las utilidades de operación (S/. 36000,00$)$ se debe básicamente a la asignación de los costos fijos de producción de S/. 180000,00 a un mayor número de unidades producidas; lo que implica que los costos fijos de producción unitarios disminuyeron de $S / .9,00$ a $S / .7,20$, es decir $S / .1,80$.

Concluyendo, el costo de los productos vendidos, cuando se producen 25000 unidades sería de S/. 1,80 menos por unidad (20000 x $1,80=36$ 000), es decir S/. 36 000,00 menos en total, que es la ganancia producida.

A diferencia del método de costeo variable, donde la utilidad no tendrá mayores cambios debido a que no importa si las cantidades producidas excedieron o no a las ventas, en este sistema no se asignan costos fijos de producción a las unidades producidas.

En cuanto al método de costeo variable, lo explicaremos en dos momentos cuando las ventas ascienden a 20000 unidades y a 25000 unidades, así apreciaremos los efectos en los ingresos operativos.

Ventas de 20000 unidades:

\begin{tabular}{|c|c|c|}
\hline \multicolumn{3}{|c|}{$\begin{array}{l}\text { Estado de Ganancias y Pérdidas por el Método del } \\
\text { Costeo Variable (Expresado en nuevos soles ) }\end{array}$} \\
\hline$(20000$ & inidades $\times$ S/. 90,00) & 1800000,00 \\
\hline $\begin{array}{l}\text { Costos variables } \\
\text { de los productos }(20000 \\
\text { vendidos }\end{array}$ & inidades x S/. 27,50) & 550000,00 \\
\hline Margen de producción & & 1250000,00 \\
\hline $\begin{array}{l}\text { Gastos variables de } \\
\text { venta y administración }\end{array}$ & $(20000 \times \mathrm{S} / .15,00)$ & $(300000,00)$ \\
\hline Margen de contribución & & 950000,00 \\
\hline \multicolumn{3}{|l|}{ Costos fijos } \\
\hline Costos fijos de producción & 300000,00 & \\
\hline Gastos fijos de ventas & 30000,00 & $(330000,00)$ \\
\hline Ingresos de operaciones & & 620000,00 \\
\hline
\end{tabular}

Ventas de 25000 unidades:

\begin{tabular}{|c|c|c|}
\hline \multicolumn{3}{|c|}{$\begin{array}{l}\text { Estado de Ganancias y Pérdidas por el Método del } \\
\text { Costeo Variable (Expresado en nuevos soles) }\end{array}$} \\
\hline Ventas & (20 000 unidades x S/. 90,00) & 1800000,00 \\
\hline $\begin{array}{l}\text { Costos variables } \\
\text { de los productos } \\
\text { vendidos }\end{array}$ & $\begin{array}{l}(25000 \text { unidades } \times \mathrm{S} / .27,50) \\
687500,00 \\
\text { Menos } 5000 \text { unidades a S/. 27,50 } \\
(137500,00)\end{array}$ & $(550000,00)$ \\
\hline \multicolumn{2}{|c|}{ Margen de producción } & 1250000,00 \\
\hline \multicolumn{2}{|c|}{$\begin{array}{l}\text { Gastos variables de } \\
\text { venta y administración }\end{array}$} & $(300000,00)$ \\
\hline \multicolumn{2}{|c|}{ Margen de contribución } & 950000,00 \\
\hline
\end{tabular}




\begin{tabular}{|lrr|}
\hline Costos fijos & & \\
\hline Costos fijos de producción & 300000,00 & \\
\hline Gastos fijos de ventas & 30000,00 & $(330000,00)$ \\
\hline Ingresos de operaciones & & 620000,00 \\
\hline
\end{tabular}

Podemos llegar a las siguiente conclusión: que cuando él numero de unidades producidas excede al numero de unidades vendidas, el ingreso por el método de costeo variable de operaciones será menor que el ingreso mediante el método de costeo absorbente.

Si las unidades vendidas, son menores a las unidades producidas, entonces, el ingreso por costeo variable es menor que el ingreso por costeo absorbente.

$\mathrm{Si}$ las unidades vendidas son mayores a las unidades producidas, entonces el ingreso por costeo variable es mayor al ingreso por costo absorbente.

El ingreso de operaciones o utilidades puede diferir bajo el método de costeo variable con respecto al método de costeo absorbente.
$\mathrm{Al}$ analizar y evaluar las operaciones, la gerencia debe estar consciente de los posibles efectos de cambiar los niveles de inventarios, bajo los dos métodos.

Cuando se utilice el método de costeo absorbente, la gerencia debe tener cuidado al analizar el ingreso de las operaciones si ocurren cambios considerables en los niveles de inventarios.

Algunos gerentes y contadores consideran que el método de costeo variable se debe utilizar para evaluar el desempeño de la operación, porque este método induce a mantener niveles de inventarios

Pero es aquí donde se debe tener mucho cuidado porque el exceso de niveles de inventarios inapropiados puede generar sobrecostos logísticos como almacenamiento y financiamiento, haciendo ineficiente $y$ financieramente inviable la cadena de suministros o cadena de valor. 
